\title{
Modeling and Design of Energy Efficient Variable Stiffness Actuators
}

\author{
L.C. Visser, R. Carloni, R. Ünal and S. Stramigioli
}

\begin{abstract}
In this paper, we provide a port-based mathematical framework for analyzing and modeling variable stiffness actuators. The framework provides important insights in the energy requirements and, therefore, it is an important tool for the design of energy efficient variable stiffness actuators. Based on new insights gained from this approach, a novel conceptual actuator is presented. Simulations show that the apparent output stiffness of this actuator can be dynamically changed in an energy efficient way.
\end{abstract}

\section{INTRODUCTION}

When robots operate in a dynamic environment, unexpected collisions between the robot and the environment are likely to occur. It is important that during these collisions, both the robot and the object it is colliding with suffer as little damage as possible. This becomes even more important when the robot operates in an environment together with humans. Human-robot collisions under several circumstances have been thoroughly investigated and it was shown that serious injury may occur due to high impact forces [1], [2]. In order to reduce the impact forces, several strategies have been proposed in [3]. One solution is to use robotic joints of which the output stiffness can be varied according to the performed task, ensuring safe human robot interaction.

Variable stiffness actuators can also play a role in energy efficient actuation, due to the presence of internal elastic elements. While actuating periodic motions, for example in locomotion, the kinetic energy can be temporarily store in the springs and, thus, reduce overall energy costs [4].

A number of variable stiffness actuators, based on different principles, have been developed. The 'Jack Spring'TM, proposed in [5], achieves a varying stiffness by changing the number of active coils of a spring. Other actuators, like AMASC [6], VSA [7], VS-Joint [8] and MACCEPA [9], rely on changing the pretension of a spring, put in series with the joint, to change the apparent joint stiffness. All these variable stiffness actuators require energy to change the stiffness, even in unloaded configurations, as is shown in [10].

A solution based on an infinite variable transmission to dynamically change the output torque, and thus the apparent stiffness, without using any energy, has been proposed in [4]. Although in theory this actuator is highly energy efficient when optimal control techniques are applied [11], currently existing infinite variable transmissions rely on friction to operate and, therefore, they inherently energy inefficient.

This work has been funded by the European Commission's Seventh Framework Programme as part of the project VIACTORS under grant no. 231554.

\{1.c.visser,r.carloni,r.unal,s.stramigioli\}@utwente.nl, IMPACT Institute, Faculty of Electrical Engineering, Mathematics and Computer Science, University of Twente, 7500 AE Enschede, The Netherlands.
In this work we present a port-based mathematical model for variable stiffness actuators. This model is derived by starting from an ideal variable stiffness joint and, then, by generalizing this concept to a generic framework that can be used to model variable stiffness actuators. This framework provides important insights in the energy requirements of the actuator and, therefore, we can derive design guidelines for realizing energy efficient variable stiffness actuators. Based on these guidelines, we present a new conceptual actuator and show that, in theory, it does not require energy to change the stiffness regardless of the state of the system.

The paper is organized as follows. In Sec. II, we formulate the problem statement in a port-based setting and present the mathematical framework. In Sec. III, we propose a novel conceptual actuator based on the analysis of the mathematical model. A control law for this actuator is presented in Sec. IV and, in Sec. V, simulation results validate the theoretical analysis. Finally, Sec. VI provides conclusions and recommendations for future work.

\section{PORT-BASED MODEL OF VARIABLE STIFFNESS ACTUATORS}

In this Section, we present a port-based mathematical model of variable stiffness actuators. We use the bond graph modeling language to visualize the concepts presented. This port-based modeling approach provides important insights to a more intuitive process for modeling, design and control of energy efficient variable stiffness actuators.

The basic idea behind the bond graph modeling language is that every physical system can be modeled by interconnecting simple elements, which are characterized by a particular port behavior [12]. The interconnections are realized through bonds, which represent the power flow between the different elements. Mathematically, the bonds are characterized by two power conjugate variables, called effort $e$ and flow $f$. If $\mathcal{F}$ is the linear space of flows, then the dual space $\mathcal{E}:=\mathcal{F}^{*}$ is the linear space of efforts. For $e \in \mathcal{E}$ and $f \in \mathcal{F}$, the dual product $\langle e \mid f\rangle$ yields power. In the mechanical domain, forces and torques are efforts, and velocities are flows.

\section{A. Problem statement}

Let $F$ be the generalized output force of a generic actuator, either a linear force in case of a linear actuator or a torque in case of rotational actuator, and $x$ the generalized output position, either a translational displacement or an angle. The stiffness $K$, sensed at the output of the actuator, is given by

$$
K=\frac{\delta F}{\delta x}
$$




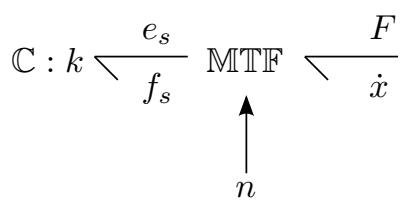

Fig. 1. Ideal variable stiffness joint - The $\mathbb{M T F}$-element is an ideal transmission, with a modulated transmission ratio $n$. The $\mathbb{C}$-element represents an ideal linear spring with a fixed stiffness $k$ and it is interconnected to the $\mathbb{M T F}$ through a bond with effort/flow $\left(e_{s}, f_{s}\right)$. The output port is characterized by effort/flow $(F, \dot{x})$ and the stiffness felt at the output port can be changed only by changing $n$.

where $\delta F$ and $\delta x$ denote infinitesimal changes in force and position, respectively. The output stiffness $K$ depends on the internal structure of the actuator (e.g. axis stiffness, presence of internal springs, etc.) and on the effects of the control action. An ideal variable stiffness actuator allows the output stiffness $K$ to be changed without using energy.

\section{B. Variable stiffness joint by using an ideal transmission}

In the ideal case, a variable stiffness joint can be realized by an ideal transmission together with a spring element. In bond graphs, it is represented as shown in Fig. 1. The $\mathbb{C}$ element represents an ideal linear spring which is characterized by an internal state $s \in \mathbb{R}$, either the compression or the elongation, a fixed stiffness $k \in \mathbb{R}$, and an internal energy $H=\frac{1}{2} k s^{2}$ stored in the spring. The port of the $\mathbb{C}$ element is characterized by the effort $e_{s}=\frac{\partial H}{\partial s}$ and the flow $f_{s}=\dot{s}$. Note that, in the figure, the direction of the half arrow determines positive power flow. The MTF- element is an ideal transmission with a modulated transmission ratio $n \in \mathbb{R}^{+}$. If we denote its two ports by effort/flow $\left(e_{s}, f_{s}\right)$ and effort/flow $(F, \dot{x})$, i.e. the force and the velocity of the output port, the relation between the two ports is given by

$$
\begin{aligned}
& F=n e_{s} \quad \rightarrow \quad F=n \frac{\partial H}{\partial s}=n k s \\
& f_{s}=n \dot{x} \quad \rightarrow \quad \dot{s}=n \dot{x}
\end{aligned}
$$

This implies that $\delta F=n k \delta s$ and $\delta s=n \delta x$. Since the $\mathbb{M T T F}$-element is power continuous, i.e. $\left\langle e_{s} \mid f_{s}\right\rangle=\langle F \mid \dot{x}\rangle$, it follows that

$$
\delta F=n^{2} k \delta x
$$

From Eqs. (1) and (3), it follows that, in the ideal case, the apparent output stiffness is given by $K=n^{2} k$. This means that, ideally, the stiffness can be modulated by only changing $n$ and without using any energy.

Note that the ideal variable stiffness joint as it is presented in this Section is passive with respect to the output port, i.e. through the output port it cannot provide more power than what is stored in the $\mathbb{C}$-element.

\section{Variable stiffness actuator}

In the purpose of realizing a variable stiffness actuator, the ideal $\mathbb{M T F}$-element has to be replaced by a non ideal transmission, as depicted in Fig. 2. The added port, characterized by generalized forces $\tau$ and generalized velocities $\dot{q}$, supplies power that can either be used to change the transmission ratio and, therefore, to change the apparent output stiffness,

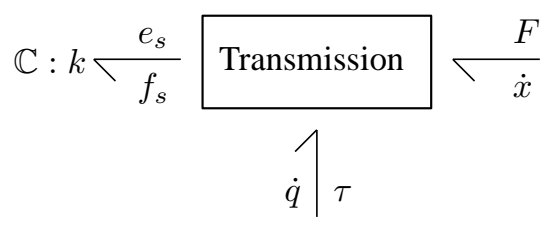

Fig. 2. Non-ideal variable stiffness actuator - The $\mathbb{C}$-element represents a linear spring with a fixed stiffness $k$ and it is interconnected to the transmission with effort/flow $\left(e_{s}, f_{s}\right)$. The output port is characterized by effort/flow $(F, \dot{x})$. The power supplied through the external port $(\tau, \dot{q})$ can be used to change the transmission ratio, and thus to change the apparent output stiffness, or to do work on the output port $(F, \dot{x})$.

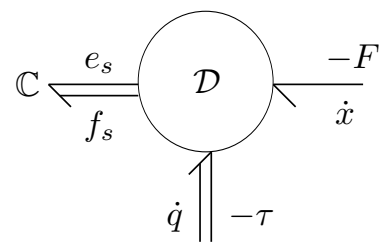

Fig. 3. Generalized representation of a variable stiffness actuator - The Dirac structure defines the interconnections between the different bonds and, therefore, how power is distributed among the ports. The multi-bonds allow any number of springs, i.e. the $\mathbb{C}$-element, and any number of external inputs $(\tau, \dot{q})$. The output port $(F, \dot{x})$ is characterized by a single-bond.

or to do work at the output port. Note that this mechanism is power continuous.

The concept of the transmission shown in Fig. 2 can be generalized by using a Dirac structure $\mathcal{D} \in\{\overline{\mathcal{D}}\}$, where $\{\overline{\mathcal{D}}\}$ is the complete set of allowable Dirac structures, i.e.,

$$
\{\overline{\mathcal{D}}\}=\{\overline{\mathcal{D}} \subset \mathcal{E} \times \mathcal{F} \mid\langle e \mid f\rangle=0 \quad \forall(e, f) \in \overline{\mathcal{D}}\}
$$

as follows from the definition in [12]. The Dirac structure is power continuous and defines the interconnection of the power ports, i.e. it describes how the power through one port is distributed to the other ports.

The generalized representation of a variable stiffness actuator through a Dirac structure is depicted in Fig. 3, in which the minus signs are to comply with its formal definition. The multi-bonds allow any number of springs, i.e. the $\mathbb{C}$ element, and any number of external inputs $(\tau, \dot{q})$. The output port $(F, \dot{x})$ is characterized by a single-bond, i.e. the output is one-dimensional. Note that the Dirac structure is not necessarily constant.

The Dirac structure of Fig. 3 can be represented by a skew-symmetric matrix $D(q, x)$ of the form

$$
\left[\begin{array}{c}
\dot{s} \\
\tau \\
F
\end{array}\right]=\underbrace{\left[\begin{array}{ccc}
0 & A(q, x) & B(q, x) \\
-A(q, x)^{T} & 0 & C(q, x) \\
-B(q, x)^{T} & -C(q, x)^{T} & 0
\end{array}\right]}_{D(q, x)}\left[\begin{array}{c}
\frac{\partial H}{\partial s} \\
\dot{q} \\
\dot{x}
\end{array}\right]
$$

where $A(q, x), B(q, x)$ and $C(q, x)$ describe the relations between the power conjugate variables of each port connected to the structure.

In the specific case of a variable stiffness actuator, we assume that

- The actuator has some internal degrees of freedom, denoted by the configuration variables $q$, which are 


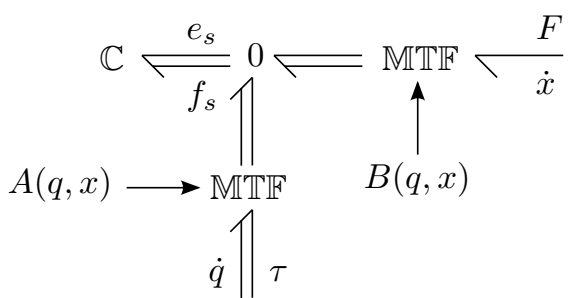

Fig. 4. Bond graph representation of the Dirac structure of a variable stiffness actuator - The MTF-elements are modulated as given by Eq. (6) and, thus, they define the power flow.

actuated through the port $(\tau, \dot{q})$.

- The apparent stiffness at the output port $(F, \dot{x})$ is related to the configuration of the internal degrees of freedom.

- Friction is neglected.

- There is no coupling between the output force $F$ and the velocities of the internal degrees of freedom $\dot{q}$, i.e. $C(q, x)=0$, because such a power continuous transformation between forces and velocities cannot be realized in the mechanical domain.

Following these assumptions, it follows

$$
\left[\begin{array}{c}
\dot{s} \\
\tau \\
F
\end{array}\right]=\underbrace{\left[\begin{array}{ccc}
0 & A(q, x) & B(q, x) \\
-A(q, x)^{T} & 0 & 0 \\
-B(q, x)^{T} & 0 & 0
\end{array}\right]}_{D(q, x)}\left[\begin{array}{c}
\frac{\partial H}{\partial s} \\
\dot{q} \\
\dot{x}
\end{array}\right]
$$

Eq. (6) can be represented in bond graphs as shown in Fig. 4.

\section{Analysis of the model}

From Eq. (6), it is easy to derive the variation of the energy stored in the system, which is given by

$$
\begin{aligned}
\frac{\mathrm{d} H}{\mathrm{~d} t} & =\frac{\partial H}{\partial s} \frac{\mathrm{d} s}{\mathrm{~d} t} \\
& =\frac{\partial H}{\partial s}(A(q, x) \dot{q}+B(q, x) \dot{x}) \\
& =-\tau^{T} \dot{q}-F^{T} \dot{x}
\end{aligned}
$$

Note that energy can be added to or removed from the system via the output port $(F, \dot{x})$ or the port $(\tau, \dot{q})$. This corresponds to the power continuity of the Dirac structure in Fig. 3.

In the purpose of designing an energy efficient variable stiffness actuator, we can state that the most energy efficient variable stiffness actuator is such that the amount of energy stored in the system does not change due to power supplied through the port $(\tau, \dot{q})$. This condition is accomplished if the term $A(q, x) \dot{q}$ in Eq. (7) is zero, i.e. if

$$
\dot{q} \in \operatorname{ker} A(q, x) \quad \forall q, x
$$

Note that taking $A=0$ reduces the system to the ideal case depicted in Fig. 1.

From this analysis, we can derive a design guideline for energy efficient variable stiffness actuators: the design of the internal configuration should include a number of internal degrees of freedom such that it is possible to change the apparent output stiffness while satisfying Eq. (8). This corresponds to decoupling position and stiffness control on

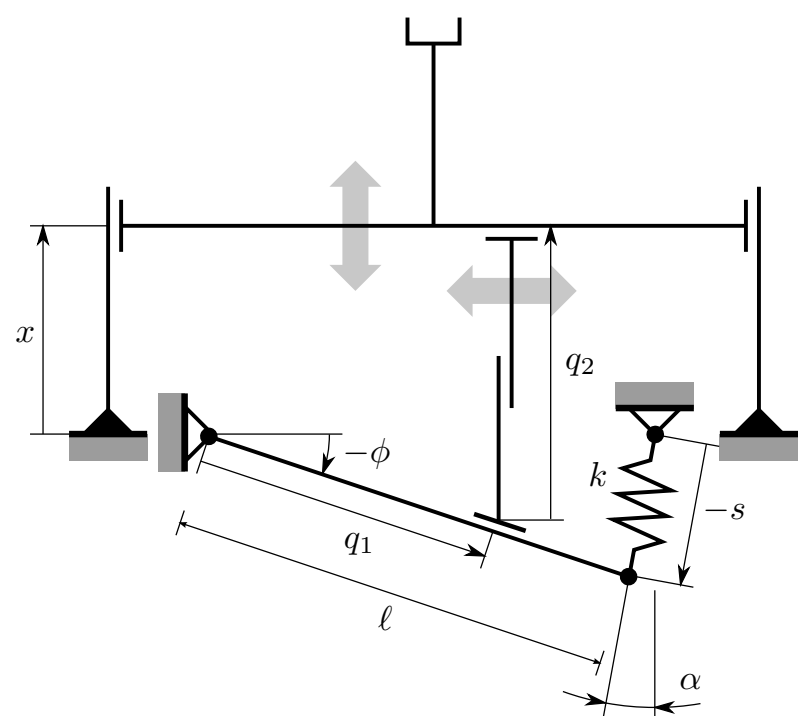

Fig. 5. An energy efficient variable stiffness actuator - The end effector is constrained to move only in $x$-direction. The internal degrees of freedom $q_{1}$ and $q_{2}$ are used to change the effective transmission ratio and to control the end effector, respectively.

a mechanical level. Due to the provided insights in power flows, the port-based framework, described by Fig. 3 and Eq. (6), provides an important tool for the design of energy efficient variable stiffness actuators.

\section{A NOVEL VARIABLE STIFFNESS ACTUATOR CONCEPT}

Following the design guideline presented in Sec. II-D, we introduce an innovative concept of a variable stiffness actuator. The design achieves a decoupling of position and stiffness control on a mechanical level, as was concluded from Eq. (8). The functional concept is based on a linear spring connected to a lever arm of variable effective length. The effective length of the lever arm determines how the stiffness of the spring is felt at the output port. Using the Dirac structure formulation in Eq. (6), we show that, by changing the internal configuration, the apparent output stiffness can be modulated without using energy.

The proposed mechanism is depicted in Fig. 5. The translational degree of freedom denoted by $q_{1}$ controls the effective transmission ratio from the spring to the end effector. It should be noted that $0<q_{1} \leq \ell$, since $q_{1}=0$ is a singular configuration in which the transmission ratio is infinite. The translational degree of freedom $q_{2}$ is used to control the end effector, which applies the force $F$ and has position $x$ (this is the output port $(F, \dot{x})$ ). Since the displacement $s$ is small compared to the lever length $\ell$, we assume $\alpha=0$.

The state $s$ of the zero free length spring is given by

$$
s=\ell \sin \phi=\ell \frac{x-q_{2}}{q_{1}}
$$

where $\phi$ is defined positive in the counter clockwise direc- 
tion. The rate of change of $s$, denoted by $\dot{s}$, is given by

$$
\begin{aligned}
\dot{s} & =\frac{\mathrm{d}}{\mathrm{d} t}\left(\ell \frac{x-q_{2}}{q_{1}}\right) \\
& =-\frac{\ell}{q_{1}}\left[\begin{array}{ll}
\sin \phi & 1
\end{array}\right]\left[\begin{array}{l}
\dot{q}_{1} \\
\dot{q}_{2}
\end{array}\right]+\frac{\ell}{q_{1}} \dot{x}
\end{aligned}
$$

From this equation it is possible to define the skew symmetric matrix which describes the Dirac structure for this particular concept. In this case, the matrix $D$ is given by

$$
\left[\begin{array}{c}
\dot{s} \\
\tau \\
F
\end{array}\right]=\underbrace{\left[\begin{array}{ccc}
0 & A(q, x) & \frac{\ell}{q_{1}} \\
-A(q, x)^{T} & 0 & 0 \\
-\frac{\ell}{q_{1}} & 0 & 0
\end{array}\right]}_{D(q, x)}\left[\begin{array}{c}
\frac{\partial H}{\partial s} \\
\dot{q} \\
\dot{x}
\end{array}\right]
$$

where

$$
A(q, x)=-\frac{\ell}{q_{1}}[\sin \phi \quad 1]
$$

The energy stored in the spring is given by the energy function $H(s)=\frac{1}{2} k s^{2}$, where $k$ is the fixed linear stiffness of the spring. Substituting Eq. (9) yields:

$$
H\left(s\left(q_{1}, q_{2}, x\right)\right)=\frac{1}{2} k\left(\ell \frac{x-q_{2}}{q_{1}}\right)^{2}
$$

The stiffness felt at the output port $K$ is given by

$$
K=\frac{\partial^{2} H}{\partial x^{2}}=\left(\frac{\ell}{q_{1}}\right)^{2} k
$$

For this particular actuator, the stiffness $K$ is only a function of $q_{1}$.

In order to have a energy efficient variable stiffness actuator, no energy should be added to or removed from the system when the stiffness is changed via the port $(\tau, \dot{q})$. For this particular system, Eq. (8) can be written as

$$
\dot{q} \in \operatorname{ker} A(q, x) \quad \forall q, x \quad \Leftrightarrow \quad \dot{q}_{2}=-\sin (\phi) \dot{q}_{1}
$$

It follows that if $\dot{q}_{1}$ is such that the stiffness $K$ changes as desired, and if $\dot{q}_{2}$ satisfies the constraint in Eq. (15), the stiffness is changed without using energy. This is valid even when the system is in a loaded configuration (i.e. $s \neq 0$ ).

It should be observed that when the stiffness is changed, the energy in the system might change. From Eq. (11) it follows that, when $q_{1}$ is changed, the force $F$ at the output port changes. If this change in force is not balanced, there is a power flow through the output port. This power flow comes from energy stored in the spring and not from the port $(\tau, \dot{q})$ since there is no power flow through the port $(\tau, \dot{q})$, i.e.

$$
\dot{q} \in \operatorname{ker} A \quad \Rightarrow \quad \tau^{T} \dot{q}=\frac{\partial^{T} H}{\partial s} A \dot{q}=0
$$

However, by expanding Eq. (16) we can notice that

$$
\begin{aligned}
\tau_{1} \dot{q}_{1} & =\frac{\partial H}{\partial s} \frac{\ell}{q_{1}} \sin (\phi) \dot{q}_{1} \\
\tau_{2} \dot{q}_{2} & =\frac{\partial H}{\partial s} \frac{\ell}{q_{1}} \dot{q}_{2}
\end{aligned}
$$

This implies that, when the stiffness is changed and Eq. (15) is satisfied, one actuator is doing positive work, while the other one is doing the same amount of negative work. If indeed two uncoupled actuators are used, the negative work is lost. Assuming that Eq. (15) is satisfied, the absolute power flow through the port $(\tau, \dot{q})$ is

$$
\begin{aligned}
|P| & =\left|\tau_{1} \dot{q}_{1}\right|+\left|\tau_{2} \dot{q}_{2}\right| \\
& =\left|\frac{\partial H}{\partial s} \frac{\ell}{q_{1}} \sin (\phi) \dot{q}_{1}\right|+\left|\frac{\partial H}{\partial s} \frac{\ell}{q_{1}} \dot{q}_{2}\right| \\
& =\left|\frac{\partial H}{\partial s} \frac{\ell}{q_{1}} \sin (\phi) \dot{q}_{1}\right|+\left|\frac{\partial H}{\partial s} \frac{\ell}{q_{1}} \sin (\phi) \dot{q}_{1}\right| \quad \text { by Eq. } \\
& =2\left|\frac{\partial H}{\partial s} \frac{\ell}{q_{1}} \sin (\phi) \dot{q}_{1}\right|
\end{aligned}
$$

It follows that the power needed to change the stiffness is proportional to $\sin \phi$ and $\dot{q}_{1}$. In the simulation results presented in Sec. V, we show that the amount of energy used to change the stiffness can be kept small.

\section{INTERNAL CONFIGURATION CONTROL}

As stated in the Introduction, in order to ensure safe operation in a dynamic environment, a moving manipulator should have a low stiffness when moving at high velocities so to reduce impact forces, while it can have a high stiffness at low velocities for increasing accuracy of motion. This approach requires that, while the end effector is moving, the output stiffness is changed. Therefore, in this Section, we derive a control law, with which the output stiffness and the end effector of the proposed variable stiffness actuator can be independently controlled. The control architecture is depicted in Fig. 6 and described in details hereafter.

\section{A. Control of the output stiffness}

From Eq. (14), it follows that the apparent output stiffness $K$ is only a function of the internal configuration $q_{1}$, i.e. $K=K\left(q_{1}\right)$. Its time derivative $\dot{K}$, i.e. the rate of change of the apparent stiffness, is then given by

$$
\dot{K}=\frac{\mathrm{d} K}{\mathrm{~d} t}=\frac{\partial K}{\partial q_{1}} \frac{\mathrm{d} q_{1}}{\mathrm{~d} t}=-2 \frac{\ell^{2} k}{q_{1}^{3}} \dot{q}_{1}
$$

This means that, if a desired stiffness profile $K_{d}$ is required and therefore a desired $\dot{K}_{d}$, the behaviour of the internal variable $q_{1}$, i.e. its desired configuration $q_{1, d}$ and its desired rate of change $\dot{q}_{1, d}$, is fixed by

$$
\begin{aligned}
& q_{1, d}=\sqrt{\frac{\ell^{2} k}{K_{d}}} \\
& \dot{q}_{1, d}=-\frac{1}{2} \frac{q_{1}^{3}}{\ell^{2} k} \dot{K}_{d}
\end{aligned}
$$

These two equations realize a feedforward control, which is denoted by $\mathrm{FF}_{q_{1}}$ in Fig. 6. The set-points $q_{1, d}$ and $\dot{q}_{1, d}$ are inputs for a PID controller, denoted with $\mathrm{PID}_{q_{1}}$ in the figure. The control output of the PID controller is directly applied to the actuator and is denoted by $\kappa_{q_{1}}$. 


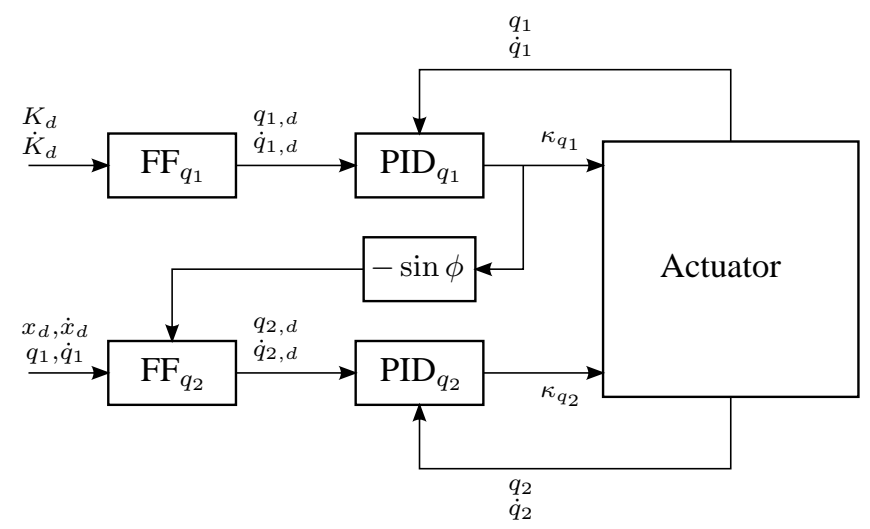

Fig. 6. Control of the internal degrees of freedom - The $\mathrm{FF}_{q_{i}}$ blocks calculate a feedforward control based on Eqs. (20) and (21), respectively. Combined with feedback PID control, this yields the control signals $\kappa_{q_{i}}$. By coupling the two controller outputs the constraint in Eq. (15) is satisfied.

\section{B. Control of the end effector position}

Let $x_{d}$ be a desired end effector position and $\dot{x}_{d}$ its time derivative. The behaviour of the internal variable $q_{2}$, i.e. its desired configuration $q_{2, d}$ and its desired rate of change $\dot{q}_{2, d}$, is fixed by

$$
\begin{aligned}
q_{2, d} & =x_{d}-\sin (\phi) q_{1} \\
\dot{q}_{2, d} & =\dot{x}_{d}-\sin (\phi) \dot{q}_{1}
\end{aligned}
$$

These two equations realize a feedforward control, which is denoted with $\mathrm{FF}_{q_{2}}$ in Fig. 6 . The set-points $q_{2, d}$ and $\dot{q}_{2, d}$ are inputs for a PID controller, denoted with $\operatorname{PID}_{q_{2}}$ in the figure. The control output of the PID is denoted by $\kappa_{q_{2}}$.

This controller satisfies the constraint Eq. (15) and thus allows the stiffness to be controlled without using energy. When the stiffness is not changed, i.e. when $\kappa_{q_{1}}=0$, the control output $\kappa_{q_{2}}$ steers the end effector to the desired position. When the stiffness is changed, the power supplied through the port $(\tau, \dot{q})$ is given by

$$
\begin{aligned}
\tau^{T} \dot{q} & =-\frac{\partial H}{\partial s} A \dot{q} \\
& =-\frac{\partial H}{\partial s} \frac{\ell}{q_{1}}\left[\begin{array}{ll}
\sin \phi & 1
\end{array}\right]\left[\begin{array}{l}
\kappa_{q_{1}} \\
\kappa_{q_{2}}
\end{array}\right]
\end{aligned}
$$

Due to the definition of the control law and if the PID controllers are properly designed, Eq. (22) shows that the power supplied through the port $(\tau, \dot{q})$ is only used to do work at the output port and no energy is used to change the stiffness. If we assume that negative work is lost, the absolute power flow through the port $(\tau, \dot{q})$ is

$$
|P|=\left|\frac{\partial H}{\partial s} \frac{\ell}{q_{1}}\left(\sin (\phi) \kappa_{q_{1}}+\kappa_{q_{2}}\right)\right|
$$

This shows again that in unloaded configurations $(\phi=$ 0 ), no energy is used to change the stiffness. In loaded configurations, the power needed to change the stiffness can be kept low by either slowly changing the stiffness (keep $\kappa_{q_{1}}$ small), or only by changing the stiffness when $\phi$ is close to zero. In both cases, the term in Eq. (23) corresponding to the power consumption for changing the stiffness can be kept

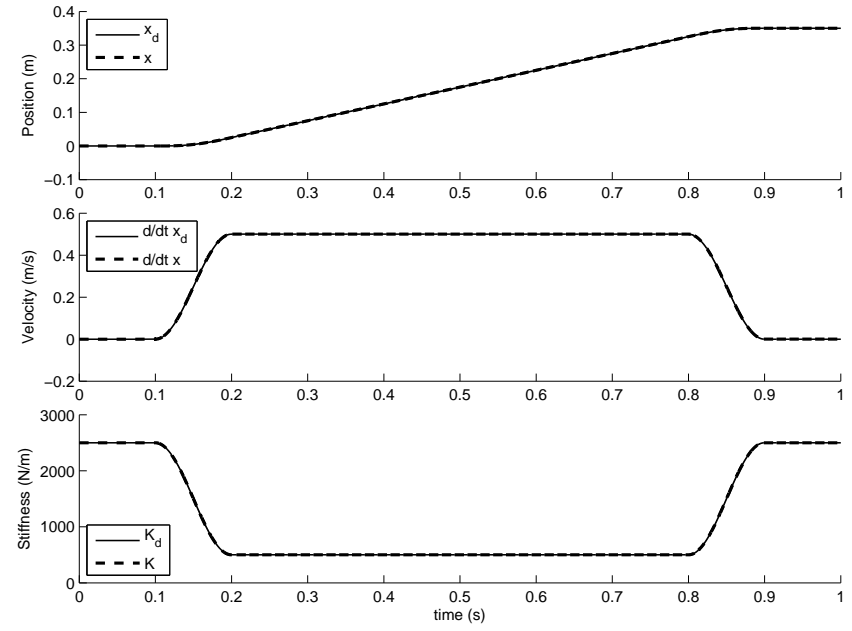

Fig. 7. Desired (continuous line) and simulated (dashed line) motion and stiffness of the actuator - While the end effector is accelerated or decelerated, the apparent output stiffness is smoothly varied to attain an appropriate level. The achieved trajectories show that the actuator can be controlled to change the stiffness and to move the end effector independently.

small compared to the power consumption for doing work at the output.

\section{SIMULATION}

To illustrate the independent control of the output stiffness and the end effector position, the following simulation experiment is performed. A mass is to be accelerated smoothly by the actuator, then moved with constant velocity and, then, smoothly decelerated to zero velocity. When the mass is not moving, the actuator stiffness should be high. When the mass is accelerated, the stiffness is to be smoothly decreased to a certain constant low value when the mass is moving at constant velocity. When the mass slows down again, the stiffness is increased. In this simulation, a mass of $1 \mathrm{~kg}$ is accelerated in $0.1 \mathrm{~s}$ from $0 \mathrm{~m} / \mathrm{s}$ to $0.5 \mathrm{~m} / \mathrm{s}$. The velocity is kept constant for $0.6 \mathrm{~s}$ and, then, the mass is decelerated at the same rate. While accelerating, the actuator stiffness is decreased from $2500 \mathrm{~N} / \mathrm{m}$ to $500 \mathrm{~N} / \mathrm{m}$. The desired trajectories are shown in Fig. 7 in continuous lines.

To control the internal degrees of freedom $q_{1}$ and $q_{2}$, the control laws proposed in Sec. IV have been implemented. The simulated end effector position and stiffness trajectories are shown in Fig. 7 in dashed lines. It can be seen that the desired trajectories are indeed followed with high accuracy.

Fig. 8 shows the energy used by each of the two actuators $q_{1}$ and $q_{2}$, the total used energy and the kinetic energy of the mass, given by $E_{\text {kin }}=\frac{1}{2} m \dot{x}^{2}$. It can be seen that all energy supplied through the port $(\tau, \dot{q})$ goes through the output port $(F, \dot{x})$ and it is stored in the mass as kinetic energy. Note that, since in Fig. 3 both ports are oriented towards the Dirac structure, i.e. positive power flows into the structure and negative power flows out of the structure, explaining the opposite signs of the plots of the kinetic energy and the actuator supplied energy. From the plots it can be seen that both $q_{1}$ and $q_{2}$ are doing negative work at some time, 


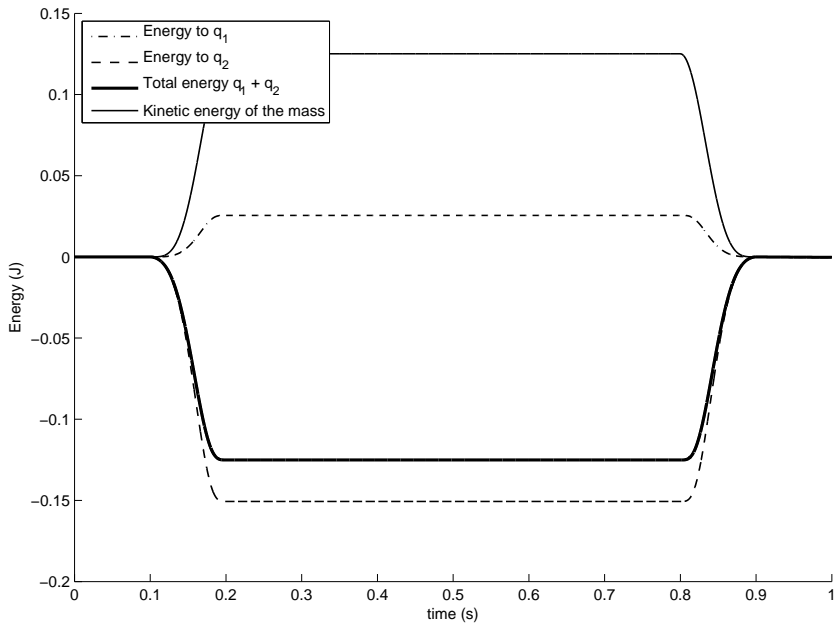

Fig. 8. Energy usage - The energy supplied by the two actuators (dashed lines) sums up (thick continuous line) to exactly the amount of kinetic energy that is stored in the mass (thin continuous line). The two energies are opposite because of convention of positive power flow in Fig. 3. Since all power supplied by the actuators is converted to kinetic energy, no energy has been used to change the stiffness.

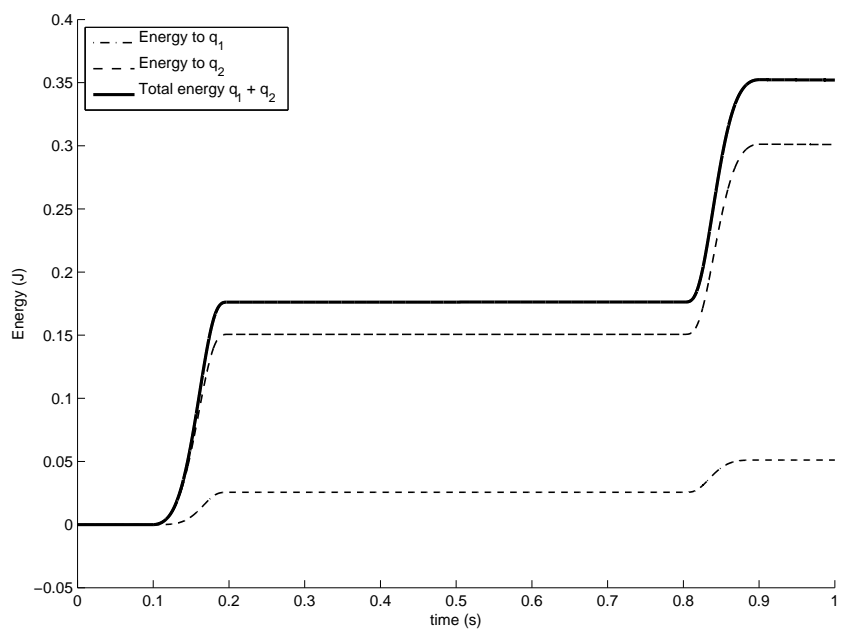

Fig. 9. Absolute energy usage - Since negative work must be considered to be lost, plotting the absolute power supplied by the actuators provides a more realistic insight in the energy usage of the actuator. It can be observed that the energy supplied by $q_{1}$ is only a fraction of the total energy supplied by the two actuators, illustrating that the actuator is energy efficient when changing stiffness.

as was described in Eq. (17). In practice, this work is lost and energy is required to change the stiffness. In Fig. 9 the absolute energy usage is shown and it can be seen that the energy used for $q_{1}$ is very small if compared to the total used energy. This is in correspondence with the argument following Eq. (23).

\section{CONCLUSIONS AND FUTURE WORK}

In this paper, we presented a mathematical framework for the analysis of variable stiffness actuators in terms of energy consumption. This framework provides conditions that should be satisfied in order to be able to change the stiffness of an actuator without using energy. Therefore, the framework is an important tool in both modeling and designing energy efficient variable stiffness actuators.

A novel variable stiffness actuator concept, which complies with the restrictions set by the mathematical framework, has been introduced. For the proposed concept, the apparent output stiffness can be changed without using energy, even in loaded conditions, but while allowing the internal degrees of freedom to do negative work. Simulation results show that the stiffness can be changed in a energy efficient way.

Future work will focus on finding performance measures for variable stiffness actuators and integrate these measures in the mathematical framework. In particular, since the presented framework provides a tool for analyzing energy efficiency of variable stiffness actuators, it is desired to derive energy efficiency measures that are closely related to the properties of the framework. These measures should give a common basis on which variable stiffness actuators can be objectively compared in terms of how energy efficient they are in changing the stiffness during actuation.

\section{REFERENCES}

[1] S. Haddadin, A. Albu-Schäffer, G. Hirzinger, "The Role of the Robot Mass and Velocity in Physical Human Robot Interaction - Part I: Nonconstrained Blunt Impacts", Proc. IEEE Int. Conf. on Robotics and Automation, 2008.

[2] S. Haddadin, A. Albu-Schäffer, G. Hirzinger, "The Role of the Robot Mass and Velocity in Physical Human Robot Interaction - Part II: Constrained Blunt Impacts", Proc. IEEE Int. Conf. on Robotics and Automation, 2008.

[3] A. Bicchi, G. Tonietti, "Fast and 'Soft-Arm' Tactics: Dealing with the Safety-Performance Tradeoff in Robot Arms Design and Control", IEEE Robotics and Automation Magazine, Vol. 11, pp. 22-33, 2004.

[4] S. Stramigioli, G. van Oort, E. Dertien, "A concept for a new Energy Efficient Actuator", Proc. IEEE/ASME Int. Conf. on Advanced Intelligent Mechatronics, 2008.

[5] K.W. Hollander, T.G. Sugar, D.E. Herring, "Adjustable Robotic Tendon using a "Jack Spring,TM", Proc. Int. Conf. on Rehabilitation Robotics, 2005.

[6] J.W. Hurst, J.E. Chestnutt, A.A. Rizzi, "An Actuator with Physically Variable Stiffness for Highly Dynamic Legged Locomotion”, Proc. IEEE Int. Conf. on Robotics and Automation, 2004.

[7] G. Tonietti, R. Schiavi, A. Bicchi, "Design and Control of a Variable Stiffness Actuator for Safe and Fast Physical Human/Robot Interaction", Proc. IEEE Int. Conf. on Robotics and Automation, 2005.

[8] S. Wolf, G. Hirzinger, "A New Variable Stiffness Design: Matching Requirements of the Next Robot Generation", Proc. IEEE Int. Conf. on Robotics and Automation, 2008.

[9] B. Vanderborght, N.G. Tsagarakis, C. Semini, R. van Ham, D.G. Caldwell, "MACCEPA 2.0: Adjustable Compliant Actuator with Stiffening Characteristic for Energy Efficient Hopping", Proc. IEEE Int. Conf. on Robotics and Automation, 2009.

[10] B. Vanderborght, R. van Ham, D. Lefeber, T.G. Sugar, K.W. Hollander, "Comparison of Mechanical Design and Energy Consumption of Adaptable, Passive-compliant Actuators", Int. Journal of Robotics Research, Vol. 28, no. 1, pp. 90-103, 2009.

[11] O. Gerelli, R. Carloni, S. Stramigioli, "Port-based Modeling and Optimal Control for a new Very Versatile Energy Efficient Actuator", IFAC Int. Symp. on Robot Control, 2009.

[12] A.J. van der Schaft, $L_{2}$-Gain and Passivity Techniques in Nonlinear Control, Springer, 2000. 